\title{
A Comparative Study Between SAM with Edema and SAM Without Edema and Associated Factors Influencing Treatment, Outcome \& Recovery
}

\author{
Zabeen Choudhury ${ }^{1, ~ *, ~ D h i m a n ~ C h o w d h u r y ~}{ }^{1}$, Tanjina Hoq ${ }^{2}$, Morjina Begum ${ }^{3}$, \\ Mohammad Shamsul Alam ${ }^{4}$ \\ ${ }^{1}$ Department of Pediatrics, Chattogram Medical College, Chattogram, Bangladesh \\ ${ }^{2}$ Department of Pediatrics, Chattogram Medical College Hospital, Chattogram, Bangladesh \\ ${ }^{3}$ Out Patient Department, Sadar Hospital, Feni, Bangladesh \\ ${ }^{4}$ Department of Forensic Medicine, North East Medical College, Sylhet, Bangladesh
}

Email address:

zabeen.chowdhury9@gmail.com (Z. Choudhury)

${ }^{*}$ Corresponding author

\section{To cite this article:}

Zabeen Choudhury, Dhiman Chowdhury, Tanjina Hoq, Morjina Begum, Mohammad Shamsul Alam. A Comparative Study Between SAM with Edema and SAM Without Edema and Associated Factors Influencing Treatment, Outcome \& Recovery. American Journal of Pediatrics. Vol. 6, No. 4, 2020, pp. 468-475. doi: 10.11648/j.ajp.20200604.24

Received: May 7, 2020; Accepted: July 10, 2020; Published: November 23, 2020

\begin{abstract}
Severe acute malnutrition (SAM) is an established contributor of under-five mortality and morbidity. Achieving desired treatment outcome has proven to be challenging. There is limited data concluding the success of treatments in the study area. Objective: This study was aimed to compare the recovery from severe acute malnutrition with identified medical complications where presence or absence of edema denotes a major predictor among children aged 0-59 months of age. Methods: This was a retrospective observational study on facility based management which was conducted in SAM block of Chattogram Medical College hospital, Chattogram, Bangladesh. Here a total of 485 patients were admitted during the period of 2013-2017 and among them, 266 patients were successfully discharged from the hospital. Based on WHO \& National guidelines for management of severely malnourished children in Bangladesh, treatment protocol, admission and discharge criteria were followed. A structured and prescribed data format was prepared and data were collected from the hospital records. Daily observation, monitoring and follow-up notes of the patients were also recorded. After data collection, they were cleaned, edited and stored into excel, EPI-INFO and analyzed by SPSS. Results: More than half of the admitted patients were cured and routinely discharged. $8.04 \%$ patients died during this period. 39.7\% (193) children recovered according to the set discharge criteria as per guidelines. Mean age of the observed patients was $22.35 \pm 15.8607$ months. More than half of the admitted patients showed moderate to good weight gain during hospital stay. Mean weight gain was higher in non-edematous patients. $50 \%$ of non - edematous patients started to gain weight in 3-5 days while $76 \%$ of edematous patients required 6-10 days to start weight gain. $4.3 \%$ patients did not gain weight during hospital stay. Both descriptive and analytic analyses were executed. $\mathrm{P}$ value $<0.05$ was considered as statistically significant Conclusions: The mean duration of hospital stay (in days) of the patients with oedema (15.64 \pm SD 7.133 days) was higher than that of the patients without oedema (9.47 \pm SD 5.881 days). But greater portion of patients with edema were cured. Independent-Sample T Test revealed the difference statistically significant, where $\mathrm{t}=(438,485)=-9.878, \mathrm{p}=0.002$.
\end{abstract}

Keywords: Severe Acute Malnutrition (SAM), Edema, MUAC, WHZ, WLZ, Weight Gain

\section{Introduction}

There are approximately 60 million children globally with MAM and 20 million with SAM. A large number of those affected are found in South Asia and Sub Saharan Africa. Around $9 \%$ of sub-Saharan African and $15 \%$ of south Asian children have moderate acute malnutrition and nearby $2 \%$ of children in developing countries have SAM. $[1,2]$ Nearly 1 to 2 million children are dying yearly because of severe acute 
malnutrition. It is reported in many poor countries that, SAM is the commonest reason for paediatric hospital admission. 25 to $30 \%$ of children with severe malnutrition die during hospital admissions. [3] In Bangladesh, SAM in children has traditionally been managed at the facility level through inpatient therapeutic care. Severely malnourished children are about 10 times at higher risk of death than those not affected. $[4,5]$ Severe acute malnutrition has two main clinical manifestations, i.e., edematous and non-edematous. However, factors associated with edema are not well established. [4] Despite recent improvement in the protocols for treatment of SAM, case-fatality rates of $20-30 \%$ are still seen and are higher for edematous malnutrition ${ }^{5}$ Over 13 million children aged less than 5 years are affected with severe acute malnutrition (SAM) in low income countries; its case fatality rate in this region is of great burden. [5] Approximately 3.1\% (BDHS 2014) of under-5 children suffering from SAM are categorized only by weight-for-length or height z-score (WHZ) $<-3$ criterion and are estimated to be a total of $\sim 450,000$. Because, there are no national information on prevalence of SAM using mid upper arm circumference (MUAC) and presence of bipedal oedema in under-5 children, thus the actual number of children suffering from SAM could be much higher than the current estimate. In children who are 6-59 months of age, WHO defines severe acute malnutrition by a very low weight-for-height/weight-for-length, or clinical signs of bilateral pitting oedema, or a very low mid-upper arm circumference. [6-8]

Oedema is a swelling caused by the accumulation of fluid in the body tissues and can be categorized as:

1. Mild (+): oedema in both feet/ankles.

2. Moderate $(++)$ : oedema in both feet plus lower legs, hands or lower arms.

3. Severe $(+++)$ : generalized oedema including both feet, legs, hands, arms and face. Children with severe acute malnutrition who have severe oedema $(+++)$ have an increased risk of mortality compared to children with severe acute malnutrition but with lesser degrees of oedema.

The standard recommendation for the management of SAM has been inpatient care in the phases of stabilization and rehabilitation. [9] Still, inpatient treatment requires many skilled and motivated staff and is resource intensive, which is often absent in low- and middle-income countries where SAM is common. [10] Besides, studies have revealed that children with SAM could be effectively treated without being admitted in hospitals. [11-14] These results have led to a brush up classification that categorizes acute malnutrition in difficult cases, which involve inpatient care in the first stage of treatment, and simple cases that can be treated in the outpatient scenery or at the community level. [15] SAM is considered less complicated if severe illness is not seen and if an adequate diet is maintained. Only children with oedema +++ or oedema $+/++$ combined with either a low MUAC, less hunger or a severe illness should be hospitalized in the first stage of the treatment regarding nutritional oedema. A recent joint statement by the World Health Organization (WHO), the United Nations Children's
Fund and the World Food Programme also advocates treating children with SAM at the community level whenever possible. [16] Though, this guidance does not distinguish grades of oedema and states generally that "children can be transferred from facility to community-based care when their oedema is reduced".

\section{Objectives}

General Objective:

To identify the outcome for the facility based management of severe acute malnutrition (SAM) children with medical complications aged 0-59 months with or without oedematous condition.

Specific Objective:

1. To identify proportions of edematous and non-edematous patients admitted with SAM.

2. To compare the socio-demographic differentials of the SAM patients with \& without edema.

3. To compare the recovery of the edematous and non-edematous SAM patients focusing on weight gain, length of hospital stay \& exit patterns.

(Therefore, this study was conducted to identify the outcome for the facility-based management of severe acute malnutrition (SAM) children with medical complication aged 0-59 months with or without oedematous condition.)

\section{Methodology and Materials}

The study was conducted in SAM block of Chittagong medical college hospital. It was from January 2013 to December 2017. The study population were all severe acute malnutrition patients aged 0-59 months with medical complication registered and admitted in the SAM block of Chattogram Medical College hospital for facility based management. A total of 485 patients were admitted in SAM block during this period. 266 of the admitted patients were successfully discharged from the hospital. Retrospective review of the hospital records of all these SAM patients were done. Here two different groups were identified: Group 1: SAM with oedema and Group 2: SAM without oedema. Secondary data of SAM was collected, analyzed and interpreted

Inclusion criteria:

Exclusion criteria (for both groups):

Children greater than 5 years of age and children less than 5 years of age who were not SAM as WHO guideline and had no medical complications \&who were not admitted in SAM block were excluded from the study.

Based on treatment protocol following admission \& discharge criteria were followed:

Management of a child with severe acute malnutrition is based on 10 essential steps which comprises stabilization phase and followed by rehabilitation phase.

These two phases has following time frame: 


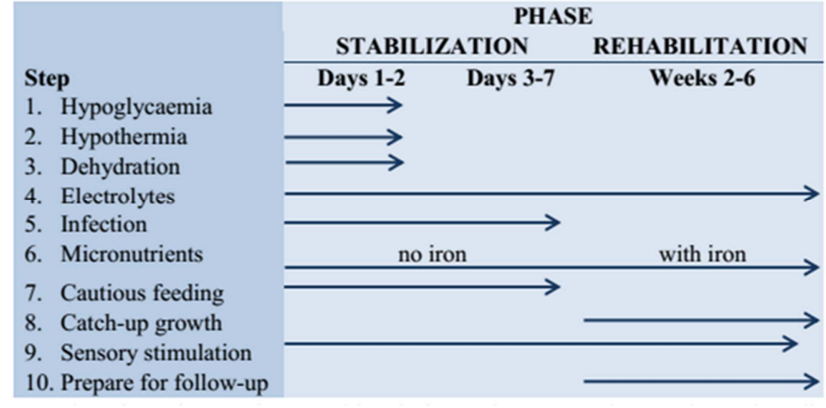

Figure 1. The time frame.

Initial phase includes identification, treatment and prevention of hypoglycaemia, hypothermia, dehydration, electrolyte imbalance, infection and management of other problems like vitamins \& micronutrient deficiencies, severe anaemia, heart failure and shock. In this phase, children are fed F-75, the locally prepared therapeutic food. Antibiotics are given according to guideline. Rehabilitation phase begins when appetite of the child returns and oedema is lost significantly. In this phase therapeutic diet for catch-up growth, F-100 and iron is introduced to the children. Stimulation of emotional \& physical development of the children and preparation of the mother/caregivers to continue home care $\&$ to know prevention of recurrence of malnutrition are being established. Patients are discharged according to the discharge criteria of the guidelines.

All retrospective data of admitted SAM patients of last consecutive five years (2013-2017) were obtained from SAM block of $\mathrm{CMCH}$ using register book database. Additionally, daily observation records including follow-up notes of clinical condition \& recovery, weight gain, prescribed drugs and all others anthropometric measurements were also recorded. The collected data was compiled and analyzed using Microsoft Excel 2010, SPSS and EPI-info. N, mean, \%s, association and other analysis were calculated by SPSS. During data collection period, the purpose and importance of the study were discussed with physicians, ethical review committee, Head and experts of pediatrics department.

\section{Results}

A total of 485 patients were admitted in SAM block during the period of 2013-2017. They were divided into three age groups. Group 1 included $<6$ months of children, group 2 included 6-24 months of children and group 3 included $>24-59$ months of children. More than one-fourth of the treated patients were admitted as oedematous malnutrition. Mean age of the patients with oedema (22.427 \pm SD 16.6677 months) was higher than that of the patients without oedema (22.323 \pm SD 15.5359 months). Assuming equal variances, to see whether this difference is statistically significant or not an independent-Sample T Test was done $\&$ it revealed the difference insignificant, where $\mathrm{t}=(438,485)=-0.065, \mathrm{p}=0.115$. Edema was found more in male patients $(86,31.0 \%)$. For statistical significance, a chi square test was done \& it revealed the difference insignificant, where $\chi^{2}(1,485)=0.758, \mathrm{df}=1, \mathrm{p}=0.384$. Greater portion of patients hailing from urban area had oedema $(53,30.1 \%)$ Patients belonging to lower class were found more edematous $(35,31.3 \%)$. A chi square test was done to see the statistical significance and revealed the difference statistically insignificant where $\chi^{2}(3,485)=0$. $0.355, \mathrm{df}=3, \mathrm{p}=0.949 .50 \%$ of non - oedematous patients started to gain weight in 3-5 days while $76 \%$ of oedematous patients required 6-10 days to start weight gain. $4.3 \%$ patients did not gain weight during hospital stay. The average weight gain was $6.8 \mathrm{~g} / \mathrm{kg} / \mathrm{day}$ or a median of $7.35 \mathrm{~g} / \mathrm{kg} /$ day. Average weight gain of the children who did not have oedema was better at the end of intervention and was $7.5491 \mathrm{~g} / \mathrm{kg} /$ day or a median of $8.7 \mathrm{~g} / \mathrm{kg} /$ day. $90 \%$ of the patients had $<3$ weeks of hospital stay. More than $60 \%$ of the admitted patients stayed up to 2 weeks in the hospital. Average stay in the nutrition block was $11.29+6.873$ days. Mean duration of hospital stay (in days) of the patients with oedema (15.64 \pm SD 7.133 days) was higher than that of the patients without oedema $(9.47 \pm$ SD 5.881 days). An independent-Sample T Test was done and the difference was found statistically significant, where $\mathrm{t}=(438,485)=-9.878$, $\mathrm{p}=0.002$. Overall more than half of the admitted patients were cured and routinely discharged. $8.04 \%$ patients died during this period. Majority of the death cases belonged to 6-24 months of age group. Septicemia \& broncho pneumonia with heart failure caused $45 \%$ of total death. Greater portion of patients having oedema were cured $(117,81.8 \%) \&$ chi square test revealed the difference statistically significant where $\chi^{2}(3,485)=61.886, \mathrm{df}=3, \mathrm{p}=0.0$.

Table 1. Total admitted patients (2013-20170).

\begin{tabular}{ll}
\hline Year & No of Patients \\
\hline 2013 & 170 \\
2014 & 139 \\
2015 & 72 \\
2016 & 64 \\
2017 & 40 \\
Total & 485 \\
\hline
\end{tabular}

Table 2. Age distribution of admitted patients ( $n=485)$.

\begin{tabular}{lll}
\hline Age in months & $\mathbf{N}$ & \% \\
\hline$<6$ & 59 & 12.16 \\
$6-24$ & 268 & 55.25 \\
$>24-60$ & 158 & 32.57 \\
\hline
\end{tabular}

Table 3. Oedematous versus non-Oedematous patients $(n=485)$.

\begin{tabular}{lll}
\hline Criteria & $\mathbf{N}$ & $\mathbf{\%}$ \\
\hline Oedematous & 143 & 29.48 \\
Non - Oedematous & 342 & 70.51 \\
\hline
\end{tabular}


Table 4. Association between respondent's oedema and age in months $(n=485)$.

\begin{tabular}{lllllll}
\hline & Presence of oedema & N & Mean & Std. deviation & df, t & P \\
\hline Age of the patient & Absent & 342 & 22.323 & 15.5359 & \multirow{2}{*}{$483,-0.065$} & $0.115^{*}$ \\
in months & Present & 143 & 22.427 & 16.6677 & \\
\hline
\end{tabular}

*Statistically not significant.

Table 5. Association between the sex of the patients and the presence of oedema $(n=485)$.

\begin{tabular}{|c|c|c|c|c|c|c|c|c|}
\hline \multirow{2}{*}{$\begin{array}{l}\text { Sex of the } \\
\text { Patient }\end{array}$} & \multicolumn{2}{|c|}{ Absence of Oedema } & \multicolumn{2}{|c|}{ Presence of Oedema } & \multicolumn{2}{|c|}{ Total } & \multirow{2}{*}{ df, $\chi^{2}$} & \multirow{2}{*}{$P$ value } \\
\hline & $\mathbf{N}$ & $\%$ & $\mathbf{N}$ & $\%$ & $\mathbf{N}$ & $\%$ & & \\
\hline Male & 191 & $69 \%$ & 86 & $31.0 \%$ & 277 & $57.1 \%$ & & \\
\hline Female & 151 & $72.6 \%$ & 57 & $27.4 \%$ & 128 & $42.9 \%$ & $1,0.758$ & $0.384 *$ \\
\hline Total & 342 & $70.5 \%$ & 143 & $29.5 \%$ & 485 & $100.0 \%$ & & \\
\hline
\end{tabular}

*Statistically not significant.

Table 6. Association between residence of the patients and the presence of oedema $(n=485)$.

\begin{tabular}{|c|c|c|c|c|c|c|c|c|}
\hline \multirow{2}{*}{$\begin{array}{l}\text { Residence of } \\
\text { the Patient }\end{array}$} & \multicolumn{2}{|c|}{ Absence of Oedema } & \multicolumn{2}{|c|}{ Presence of Oedema } & \multicolumn{2}{|l|}{ Total } & \multirow{2}{*}{ df, $\chi^{2}$} & \multirow{2}{*}{$P$ value } \\
\hline & Frequency & Percentage & Frequency & Percentage & Frequency & Percentage & & \\
\hline Rural & 219 & $70.9 \%$ & 90 & $29.1 \%$ & 309 & $63.7 \%$ & & \\
\hline Urban & 123 & $69.9 \%$ & 53 & $30.1 \%$ & 176 & $36.3 \%$ & $1,0.053$ & $0.819^{*}$ \\
\hline Total & 342 & $70.5 \%$ & 143 & $29.5 \%$ & 485 & $100.0 \%$ & & \\
\hline
\end{tabular}

*Statistically not significant.

Table 7. Association between socio-economic status of the patients and the presence of oedema $(n=485)$.

\begin{tabular}{llllllll}
\hline \multirow{2}{*}{$\begin{array}{l}\text { Socio-economic } \\
\text { status of the Patient }\end{array}$} & \multicolumn{2}{l}{ Absence of Oedema } & Presence of Oedema & Total & \multirow{2}{*}{ df, $\boldsymbol{\chi}^{2}$} & \multirow{2}{*}{ P value } \\
\cline { 2 - 6 } & Frequency & Percentage & Frequency & Percentage & Frequency & Percentage & \\
\hline Lower Class & 77 & $68.8 \%$ & 35 & $31.3 \%$ & 112 & $23.1 \%$ & $3,0.355$ \\
Lower middle class & 165 & $70.5 \%$ & 69 & $29.5 \%$ & 234 & $48.2 \%$ & $0.949 *$ \\
Upper middle class & 91 & $72.2 \%$ & 35 & $27.8 \%$ & 126 & $26.0 \%$ & \\
Upper Class & 9 & $69.2 \%$ & 4 & $30.8 \%$ & 13 & $2.7 \%$ & \\
Total & 342 & $70.5 \%$ & 143 & $29.5 \%$ & 485 & $100.0 \%$ & \\
\hline
\end{tabular}

*Statistically not significant.

Table 8. Days required to start weight gain $(n=464)$

\begin{tabular}{lllll}
\hline Days & Non-Oedematous patient (\%) & Oedematous patient (\%) & Total & \% \\
\hline $3-5$ & $167(50.9)$ & $9(6.6)$ & 176 & 37.9 \\
$6-10$ & $146(44.5)$ & $104(76.4)$ & 250 & 52.8 \\
$>10$ & $15(4.8)$ & 38 & 8.1 & \\
\hline
\end{tabular}

Table 9. Weight gain pattern of admitted patients.

\begin{tabular}{lll}
\hline Wt. gain $(\mathbf{g m} / \mathbf{k g} / \mathbf{d a y})$ & No. of patients & Percentage \\
\hline$<5$ & 163 & 33.6 \\
$5-10$ & 151 & 31.13 \\
$>10$ & 171 & 35.25 \\
\hline
\end{tabular}

Table10. Duration of hospital stay of the patients ( $n=485$ ).

\begin{tabular}{lll}
\hline Duration (week) & $\mathbf{N}$ & \% \\
\hline$<1$ & 146 & 30.10 \\
$1-2$ & 187 & 38.55 \\
$>2-3$ & 106 & 21.85 \\
$>3$ & 46 & 9.48 \\
\hline
\end{tabular}

Table 11. Association between respondent's oedema and duration of hospital stay in days ( $n=485)$.

\begin{tabular}{lllllll}
\hline & Presence of oedema & $\mathbf{N}$ & Mean & Std. deviation & df, $\mathbf{t}$ & P \\
\hline Duration of hospital & Absent & 342 & 9.47 & 5.881 & \multirow{2}{*}{$483,-9.878$} & $0.002^{*}$ \\
stay in days & Present & 143 & 15.64 & 7.133 & \\
\hline
\end{tabular}

\footnotetext{
*Statistically significant.
} 
Table 12. Exit pattern of admitted patients ( $n=485)$.

\begin{tabular}{lllll}
\hline Year & Cured & Death & Defaulter & Others \\
\hline 2013 & 91 & 19 & 51 & 09 \\
2014 & 76 & 07 & 50 & 06 \\
2015 & 37 & 05 & 21 & 09 \\
2016 & 44 & 04 & 11 & 05 \\
2017 & 18 & 04 & 16 & 02 \\
Total & 266 & 39 & 149 & 31 \\
Percentage & 54.84 & 8.04 & 30.72 & 6.39 \\
\hline
\end{tabular}

Table 13. Association between presence of oedema and outcome of management $(n=485)$.

\begin{tabular}{|c|c|c|c|c|c|c|c|c|c|c|c|c|}
\hline \multirow{2}{*}{$\begin{array}{l}\text { Presence of } \\
\text { oedema }\end{array}$} & \multicolumn{2}{|c|}{ Death } & \multicolumn{2}{|c|}{ Defaulter } & \multicolumn{2}{|c|}{ Cured } & \multicolumn{2}{|c|}{ Others } & \multicolumn{2}{|c|}{ Total } & \multirow{2}{*}{ df, $\chi^{2}$} & \multirow{2}{*}{$\begin{array}{l}P \\
\text { value }\end{array}$} \\
\hline & $\mathbf{N}$ & $\%$ & $\mathbf{N}$ & $\%$ & $\mathbf{N}$ & $\%$ & $\mathbf{N}$ & $\%$ & $\mathbf{N}$ & $\%$ & & \\
\hline Absent & 29 & $8.5 \%$ & 129 & $37.7 \%$ & 153 & $44.7 \%$ & 31 & $9.1 \%$ & 342 & $63.7 \%$ & & \\
\hline Present & 10 & $7 \%$ & 16 & $11.2 \%$ & 117 & $81.8 \%$ & 0 & $0 \%$ & 176 & $36.3 \%$ & $3,61.886$ & $0.0^{*}$ \\
\hline Total & 39 & $8 \%$ & 145 & $29.9 \%$ & 270 & $55.7 \%$ & 31 & $6.4 \%$ & 485 & $100.0 \%$ & & \\
\hline
\end{tabular}

Table 14. Age distribution of death cases $(n=39)$.

\begin{tabular}{lll}
\hline Age (month) & N & \% \\
\hline$<6$ & 7 & 17.94 \\
$6-24$ & 28 & 71.79 \\
$>24-60$ & 4 & 10.25 \\
\hline
\end{tabular}

Table 15. Causes of death of the participants $(n=39)$.

\begin{tabular}{lll}
\hline Causes of death & N & \% \\
\hline Septicemia & 10 & 25.64 \\
Bronchopneumonia with Heart failure & 09 & 23.07 \\
Congenital Heart disease with Bronchopneumia & 05 & 12.82 \\
Encephalitis & 03 & 7.69 \\
Acute gastroenteritis with Dyselectrolytaemia & 02 & 5.12 \\
Acute Bacterial Meningitis & 02 & 5.12 \\
Severe anemia with Heart failure & 03 & 7.69 \\
CP with Aspiration pneumonia & 02 & 5.12 \\
Measles with Encephalitis & 01 & 2.56 \\
Disseminated TB & 01 & 2.56 \\
Tubercular Meningitis & 01 & 2.56 \\
\hline
\end{tabular}

Table 16. Cross tabulation Presence of oedema versus Outcome of management ( $(n=485)$.

\begin{tabular}{|c|c|c|c|c|c|c|c|}
\hline & & & \multicolumn{4}{|c|}{ Outcome of management } & \multirow{2}{*}{ Total } \\
\hline & & & Death & Defaulter & Cured & Others & \\
\hline \multirow{8}{*}{$\begin{array}{l}\text { Presence of } \\
\text { oedema }\end{array}$} & \multirow{4}{*}{ Absent } & Count & 29 & 129 & 153 & 31 & 342 \\
\hline & & $\%$ within Presence of oedema & $8.5 \%$ & $37.7 \%$ & $44.7 \%$ & $9.1 \%$ & $100.0 \%$ \\
\hline & & $\%$ within Outcome of management & $74.4 \%$ & $89.0 \%$ & $56.7 \%$ & $100.0 \%$ & $70.5 \%$ \\
\hline & & $\%$ of Total & $6.0 \%$ & $26.6 \%$ & $31.5 \%$ & $6.4 \%$ & $70.5 \%$ \\
\hline & \multirow{4}{*}{ Present } & Count & 10 & 16 & 117 & 0 & 143 \\
\hline & & $\%$ within Presence of oedema & $7.0 \%$ & $11.2 \%$ & $81.8 \%$ & $0.0 \%$ & $100.0 \%$ \\
\hline & & $\%$ within Outcome of management & $25.6 \%$ & $11.0 \%$ & $43.3 \%$ & $0.0 \%$ & $29.5 \%$ \\
\hline & & $\%$ of Total & $2.1 \%$ & $3.3 \%$ & $24.1 \%$ & $0.0 \%$ & $29.5 \%$ \\
\hline \multirow{4}{*}{ Total } & & Count & 39 & 145 & 270 & 31 & 485 \\
\hline & & $\%$ within Presence of oedema & $8.0 \%$ & $29.9 \%$ & $55.7 \%$ & $6.4 \%$ & $100.0 \%$ \\
\hline & & $\%$ within Outcome of management & $100.0 \%$ & $100.0 \%$ & $100.0 \%$ & $100.0 \%$ & $100.0 \%$ \\
\hline & & $\%$ of Total & $8.0 \%$ & $29.9 \%$ & $55.7 \%$ & $6.4 \%$ & $100.0 \%$ \\
\hline
\end{tabular}

*Statistically significant.

\section{Discussion}

Maximum studies on severe acute malnutrition have focused on children under the age of five years. In previous studies, severe malnutrition were defined in children aged between 6-59 months [17] as weight-for height (or length) less than $-3 \mathrm{Z}$-score, or less than $70 \%$ of the median National Center for Health Statistics (NHCS)/WHO reference values, or the presence of oedematous malnutrition. An orderly review was conducted to observe admission and discharge criteria for severe acute malnutrition in children who are 659 months old. [18] The search recognized 11 relevant epidemiological studies. Three of the 11 studies used cut-off values for admission that did not resemble to the WHO definition of severe acute malnutrition, namely mid-upper arm circumference $<120 \mathrm{~mm}$, mid-upper arm circumference 
$<130 \mathrm{~mm}$ and weight-for-height $<-2$ Z- score.[19-21] In the present study, a total of 485 patients were admitted in SAM block during January 2013- December 2017. Among them, 266 patients were successfully discharged from the hospital. Number of admitted patients was comparatively low during last 3 years (2015-2017). Good motivation of parents \& caregivers focusing the need of minimum 3 weeks hospital stay in SAM block may overcome such low admission. More than one-fourth of the treated patients were admitted as edematous malnutrition. Among three age groups, more than half of the admitted patients belonged to $6-24$ months of age. Mean age \pm SD was $22.354 \pm 15.8607$ months. Mean age of edematous patients was insignificantly higher than that of non-edematous patients. Weaning of the babies starts from 6 months of age and continues up to 24 months. So, improper weaning may contribute development of malnutrition in this age group. Overall male patients were admitted more and also greater portion of male patients had edema during admission. Though majority of the admitted patients came from rural area, greater portion of urban patients had edema. Working mother, lack of loving caregivers, overcrowded slum living, unhealthy food hygiene etc. might contributed such difference which was not statistically significant. Patients of lower class were found to have more edema (35, $31.3 \%$ ). More than half of the admitted patients showed moderate to good weight gain during hospital stay. Weight gain $>10 \mathrm{~g} / \mathrm{kg} /$ day is considered good, $5-10 \mathrm{~g} / \mathrm{kg} /$ day is moderate and $<5 \mathrm{~g} / \mathrm{kg} /$ day is poor. The median weight gain of the children at SAM block during the clinical review \& evaluation was $7.35 \mathrm{~g} / \mathrm{kg} /$ day and is considered to be moderate. At the same time about one-third of these children were having poor weight gain. When considering non-oedematous children, the median weight gain was $8.7 \mathrm{~g} / \mathrm{kg} /$ day which is also considered to be moderate. Frequent monitoring of all aspects of feeding \& adequacy of multivitamin, addressing untreated infection including HIV \& behavioral problems may help to improve weight gain. More than three-fourth of oedematous patients required 6-10 days to start weight gain whereas half of the non oedematous patients started to gain weight in 3-5 days. Edematous patients at first lost edema fluid and then gained weight. $4.3 \%$ patients did not gain weight during hospital stay. $90 \%$ of the patients had $<3$ weeks of hospital stay. Average stay in the nutrition block was $11.29+6.873$ days. Mean duration of hospital stay of the edematous patients was higher than that of the non-edematous patients. Edematous patients recover slow as they have more complications. They usually present late as malnutrition as weight gain due to edema often mislead the caregivers initially. An orderly review inspected the sign on the success of supervision children who are more than 6 months of age with basic oedematous malnutrition grade $+/++, 1$ in ambulatory settings. [22] Eight reports of them defined consequences of children with SAM who had bilateral oedema and who were treated as outpatients. The data were from case-series that did not have control groups and so comparisons were not therefore possible. Among basic cases of oedematous malnourished children, recovery rates were more than $88 \%$ and case-fatality rates remained below $4 \%$. Classification of oedema varied among the studies and in some studies, it was not clear if children with severe oedema were left out from outpatient treatment. These outcomes are consistent with those recommended in the Sphere guidelines and the Prudhon index for case-fatality rates. [23, 24] Authors recommended that this was because of sharing ready-to-use therapeutic food at home or lacking of food supplementation in the home-based treatment group. [25] Procedures to evaluate weight gain were not, however, uniform across the studies. No connection was reported between the severity of oedema and the likelihood of nutritional recovery or not. More than half of the admitted patients in the present study were cured and routinely discharged. 39.7\% (193) children recovered according to the set discharge criteria as per guidelines. Besides, another 73 patients were also discharged as per request of the caregivers as they had some unavoidable purposes to be served at home; they were clinically well and were also discharged with advices. Their general condition was presumed to be satisfactory for discharge. So they were also considered as routine discharged though they did not achieve the target body weight for discharge. Accumulating both of these groups of patients, a total of $266(54.84 \%)$ children were routinely discharged from the unit. The 2009 joint United Nations statement projected using a single discharge criterion of $15 \%$ (or $20 \%$, depending on the local context) weight gain over oedema-free weight on enrolment, for children admitted based on weight-for-height or mid-upper arm circumference, as well as absence of oedema for 2 weeks.[26] Majority of the death cases in the current study belonged to 6-24 months of age group. Thirty nine (39) patients died with a case-fatality rate of $8.04 \%$ during the review. There was not sufficient information to conclude whether the deaths could be avoided or not. But it was revealed that all the death cases were complicated due to the poor nutritional status of the children.

\section{Conclusion and Recommendations}

The findings of the study revealed that hospital stay of oedematous SAM patients was higher than that of the non-edematous SAM patients. The mean duration of hospital stay (in days) of the patients with oedema (15.64 \pm SD 7.133 days) was higher than that of the patients without oedema (9.47 \pm SD 5.881 days). This difference is statistically significant The overall recovery rate was $55.73 \%$ where $81.8 \%$ children recovered from SAM with the presence of oedema and $44.7 \%$ without the presence of oedema. Special focus should be given on other predictors of recovery rate like administering routine antibiotics, prompt and appropriate management of co-morbidities and counseling \& motivating mothers. Caregivers should be positively motivated before entry in the SAM block \& such motivation should be continued during the hospital stay. The average weight gain $(6.8 \mathrm{~g} / \mathrm{kg} / \mathrm{day})$ and the defaulter rate $(29.9 \%)$ were below acceptable standards. Some causes of such high defaulter rate could be assumed on 
discussing with the working doctors \& nurses like financial loss as being working mothers/caregivers, family problems, other kids at home, slow improvement of the patient, new complications etc. They did not continue the treatment in the hospital. Death rate was also considered modearte. Further prospective studies are recommended using organized, uniform and informative admission forms that are easy to fill up for better record keeping. Additionally updated discharge forms for overall progress visualization and provision of early reviewing $\&$ analysis of death cases must be included in such studies. Counseling and follow-up sessions are also possible to enroll in these prospective studies. All of these together will better reflect the performance of such nutrition units.

\section{Limitations of the Study}

The study has some limitations. Number of the admitted patients were not uniform. Hospital records of all the patients were also not uniform. Follow-up notes were incomplete in some patients. Regarding outcome, defaulter rate and death rate were high. A total of 149 (30.72\%) patients left against medical advice before completion of the treatment or recovery of the illness. The causes of premature leave from the nutrition block could not be revealed from hospital records as the causes were not recorded. These defaulted bulk might affected the case fatality rate.

\section{References}

[1] The republic of Uganda Ministry of Health Integrated Management of Acute Malnutrition Guidelines Kampala (2010).

[2] Yebyo HG, Kendall C, Nigusse D, Lemma W (2013) Outpatient Therapeutic Feeding Program Outcomes and Determinants in Treatment of Severe Acute Malnutrition in Tigray, Northern Ethiopia: A Retrospective Cohort Study. PloS one 8: e65840.

[3] WHO, UNICEF, WFP (2007) Community-based management of severe acute malnutrition? Geneva, Switzerland.

[4] WHO Guideline: Updates on the management of severe acute malnutrition in infants and children. Geneva, World Health Organization; 2013.

[5] National facility based inpatient care guideline $3^{\text {rd }}$ edition June 2017.

[6] World Health Organization, World Food Program, United Nations System Standing Committee on Nutrition and United Nations Children's Fund (2007). Community-based management of severe acute malnutrition. Geneva: World Health Organization; Rome: World Food Program; Geneva: United Nations System Standing Committee on Nutrition; New York: United Nations Children's Fund.

[7] FMOH (2007). Protocol for the management of severe acute malnutrition. Addis Ababa, Ethiopia: FMOH.

[8] WHO (2013)? Guideline: updates on the management of severe acute malnutrition in infants and children. Geneva: World Health Organization.
[9] Management of severe malnutrition: a manual for physisians and other senior health workers. Geneva, WHO, 1999.

[10] Collins S et al. Management of severe acute malnutrition in children. Lancet, 2006, 368: 1992-2000.

[11] Linneman $\mathrm{Z}$ et al. A large-scale operational study of home-based therapy with ready-to-use therapeutic food in childhood malnutrition in Malawi. Maternal \& Child Nutrition, 2007, 3: 206-215.

[12] Ciliberto MA et al. Home-based therapy for oedematous malnutrition with ready-to-use therapeutic food. Acta Paediatrica, 2006, 95: 1012-1015.

[13] Sandige $\mathrm{H}$ et al. Home-based treatment of malnourished Malawian children with locally produced or imported ready-to-use food. Journal of Pediatric Gastroenterology and Nutrition, 2004, 39: 141-146.

[14] Gaboulaud V et al. Could nutritional rehabilitation at home complement or replace centre-based therapeutic feeding programmes for severe malnutrition? Journal of Tropical Pediatrics, 2007, 53: 49-51.

[15] Collins S et al. Key issues in the success of community-based management of severe malnutrition. Food and Nutrition Bulletin, 2006, 27: S49-S82.

[16] A Joint Statement by the World Health Organization, the World Food Programme, the United Nations System Standing Committee on Nutrition and the United Nations Children's Fund. Community-based management of severe acute malnutrition. Geneva, WHO/WFP/UN/UNICEF, 2007.

[17] Caulfield LE, De Onis M, Blössner M, Black RE: Undernutrition as an underlying cause of child deaths associated with diarrhea, pneumonia, malaria, and measles. Am J Clin Nutr 2004, 80 (1): 193-198.

[18] Roberfroid D et al. Background review. Utilization of mid-upper arm circumference versus weight-for-height in nutritional rehabilitation programmes. 2013.

[19] Bekele Y, Goossens S, Harczi G, Minetti A, Ouannes M, Loretxu P. Activity report of a communitybased therapeutic feeding program with MUAC as exclusive admission criteria. Geneva: Médecins sans Frontieres; 2010.

[20] Nielsen J Valentiner-Branth P, Martins C, Cabral F, Aaby P. Malnourished children and supplementary feeding during the war emergency in Guinea-Bissau in 1998-1999. Am. J. Clin. Nutr. 2004; 80: 1036-42.

[21] Ciliberto $\mathrm{M}$ et al. Comparison of home-based therapy with ready-to-use therapeutic food with standard therapy in the treatment of malnourished Malawian children: a controlled, clinical effectiveness trial. Am. J. Clin. Nutr. 2005, 81: 86470.

[22] Roberfroid D et al. Background review. Management of oedematous malnutrition in infants and children aged $>6$ months: a systematic review of evidence. 2013 (www.who.int/nutrition/publications/guidelines/updates_mana gement_SAM_infantandchildren_reviewpaper2.pdf).

[23] The Sphere handbook - humanitarian charter and minimum standards in humanitarian response. Bourton on Dunsmore: The Sphere Project; 2011 (http://www.sphereproject.org/re sources). 
[24] Prudhon C, Golden MH, Briend A, Mary JY. A model to standardize mortality of severely malnourished children using nutritional status on admission to therapeutic feeding centres. Eur. J. Clin. Nutr. 1997; 51: 771-7.

[25] Khanum S, Ashworth A, Huttly S. Controlled trial of three approaches to the treatment of severe malnutrition. Lancet, 1994; 344: 1728-32.
[26] WHO child growth standards and the identification of severe acute malnutrition in infants and children. A joint statement by the World Health Organization and the United Nations Children's Fund. Geneva: World Health Organization; 2009. 